\title{
Percepción de criterios de uso, selección y cementación de postes intrarradiculares en rehabilitación post endodóntica
}

\section{Perception of criteria for use, selection and cementation of intrarradicular posts in post endodontic rehabilitation}

\author{
David Ugarte-Mamani ${ }^{1}$, Sively Mercado-Mamani ${ }^{2}$,Rildo Tapia-Condori ${ }^{3 *}$, Luz Mamani-Cahuata $^{4}$. \\ ${ }^{1}$ Facultad de odontología, Universidad Andina Néstor Cáceres Velásquez. Juliaca, Perú. \\ ${ }^{2}$ Especialista en Ortodoncia y ortopedia maxilar, Doctora en Salud Pública. \\ ${ }^{3}$ Especialista en Endodoncia. \\ ${ }^{4}$ Facultad de odontología, Universidad Nacional del Altiplano, Perú. \\ *dugartepoo188@gmail.com
}

\begin{abstract}
Resumen
Objetivos: Evaluar la percepción del conocimiento y la experiencia clínica, y obtener información científica y documentada de los criterios que se manejan en la región altoandina del Perú en la rehabilitación post endodóntica. Materiales y métodos: Se realizó un estudio transversal utilizando un instrumento validado con una población de odontólogos ( $\mathrm{n}=100)$ en la ciudad de Juliaca-Perú, realizándose previamente una prueba piloto con un $20 \%$ de la población. Se midieron variables tales como criterios dentales, criterios clínicos y criterios de los postes intrarradiculares. Se utilizaron estadística descriptiva y la prueba de chi cuadrado (estadística inferencial para variables cualitativas).Resultados: Un 68,8\% utiliza los postes colados de acuerdo a la forma de obtención y el material, un $93,6 \%$ siempre utiliza postes colados en ausencia de estructura coronal remanente; un $61 \%$ considera el efecto férula de $2 \mathrm{~mm}$ previo a la rehabilitación; para un $53 \%$ el costo influye a la hora de elegir el tratamiento; un $78 \%$ desconoce otros sistemas de rehabilitación post endodoncia. El sistema de cementación para los postes colados es el ionómero de vidrio $57,8 \%$, y para los postes de fibra de vidrio el cemento dual $57,8 \%$. En todos los casos p $<0,05$ se consideró significativo.Conclusiones: Los criterios evaluados tienen gran validez científica puesto que aportan el nivel de conocimiento que maneja la población altoandina en relación a diferentes estudios a nivel mundial.
\end{abstract}

Palabras clave: Canal radicular, rehabilitación bucal, restauración, postes.

\begin{abstract}
Aim: To evaluate the perception of knowledge and clinical experience, and obtain scientific and documented information on the criteria used in the high Andean region of Peru in post-endodontic rehabilitation. Material and methods: A cross-sectional study was conducted using a validated instrument with a population of dentists $(n=100)$ in the city of Juliaca-Peru, previously carrying out a pilot test with $20 \%$ of the population. Variables such as dental criteria and intraradicular posts criteria were measured. Descriptive statistics and the chi-squared test were used (inferential statistics for qualitative variables).Results: $68.8 \%$ use the cast posts according to the way of obtaining and the material, 93.6\% always use cast posts in the absence of remaining coronal structure; $61 \%$ consider the splinting effect of $2 \mathrm{~mm}$ prior to rehabilitation; for $53 \%$ the cost influences when choosing the treatment; $78 \%$ are unaware of other post-endodontic rehabilitation systems. The cementing system for the cast posts is the $57.8 \%$ glass ionomer, and for the fiberglass poles the $57.8 \%$ dual cement. In all cases $p<0.05$ was considered significant. Conclusions: The criteria evaluated have great scientific validity since they provide the level of knowledge that the Andean population handles in relation to different studies worldwide.
\end{abstract}

Key words: Root canal, oral rehabilitation, restoration, posts.

\section{INTRODUCCIÓN}

La rehabilitación de un diente tratado endodónticamente difiere de muchos factores para su posterior rehabilitación, existiendo el problema de que los odontólogos utilizan un sistema de postes adecuados para cada realidad del paciente y en especial del diente, pero cuál es el mejor sistema que debería ser aplicado en dientes tratados endodónticamente.

Un resultado clínico exitoso de los dientes tratados endodónticamente, depende de una adecuada instrumentación de conducto $^{1,2}$ y obturación tridimensional, ${ }^{3,4}$ así como en el tratamiento de restauración 
adecuado realizado después. ${ }^{5}$

La pérdida de más de la mitad de la estructura coronal del diente, causado por la caries, fractura o extensa preparación de la cavidad, exige el uso de postes, ${ }^{6}$ ya que proporcionan restauraciones con retención y estabilidad mejorada. ${ }^{7}$

De acuerdo con las conclusiones de un estudio realizado por Dietschi et al., en la restauración de los dientes tratados endodónticamente el enfoque ha evolucionado a partir de un nivel totalmente empírica para la aplicación actual de los conceptos biomecánicos para guiar el proceso de toma de decisiones. ${ }^{8}$ En primer lugar, la preservación de tejido dental, la presencia de un efecto de férula, y la adhesión se consideran las condiciones más eficaces para el éxito a largo plazo del procedimiento de restauración. ${ }^{8,9}$

En los últimos años, un mayor interés se ha visto en el enfoque de la investigación basada en la práctica dental, donde se toman las preferencias de los dentistas en consideración y los tratamientos se evaluaron en un "mundo real. ${ }^{\text {es }}$ cenario de la práctica clínica. ${ }^{10,11}$ Esta forma de asociar el conocimiento con la interpretación científica ha sido considerada el mejor método, y puede ser implementado directa y rápidamente en la práctica clínica habitual. ${ }^{12}$

El objetivo de este estudio fue evaluar los criterios de uso, selección y cementación de postes intrarradiculares en la rehabilitación post endodóntica; ya que muchos consideran que el nivel de conocimiento es muy distinto por parte de los profesionales, pero que se caracteriza por la universalidad de conocimientos a nivel mundial; siendo de caracterización la uniformidad de los usos de los sistemas intrarradiculares.

\section{MATERIALES Y MÉTODOS}

Se realizó un estudio transversal en el sur del Perú en la ciudad de Juliaca a $3.825 \mathrm{msnm}$, durante el periodo de agosto a noviembre de 2016.

La población de estudio consistió en un grupo de odontólogos de la ciudad de Juliaca, en el departamento de Puno. Se calculó el tamaño de la muestra utilizando la fórmula para estimar proporciones.

En una población finita con un margen de error de $5 \%$ y un intervalo de confianza del $95 \%$. Se evaluó a un total de 100 odontólogos de la ciudad de Juliaca distribuidos en un $55 \%$ masculino, $45 \%$ femenino. Siendo estos los que cumplían criterios de inclusión de profesionales con años de experiencia mayores a dos años teniendo un intervalo de: 2 a 5 años un $61 \%$, de 6 a 10 años con $16 \%$ y un $23 \%$ mayor a 11 años de experiencia clínica.
El estudio fue aprobado por el comité de ética local de la Facultad de Odontología de la Universidad Andina Néstor Cáceres Velásquez de la ciudad de Juliaca del departamento de Puno.

La percepción de los criterios de uso, selección y cementación se evaluó en base a conocimientos teóricos, con preguntas como ¿en caso de no existir estructura remanente Ud. usa? Postes colados (siempre, algunas veces, nunca). Las preguntas formuladas estuvieron basadas a las variables tales como:

a) Criterios dentales: la consideración de la presencia del efecto férula en la estructura a restaurar; ausencia de estructura coronal remanente. La diferencia tanto en el sector anterior y posterior para lo cual se utilizó la clasificación de Murgueito. $^{13}$

b) Criterios de los postes intrarradiculares: de acuerdo a la técnica de obtención y al tipo de material del poste, sistema de cementación.

c) Criterios clínicos: la experiencia de fractura en los postes colados y prefabricados (fibra de vidrio); la influencia que representa el costo y el conocimiento de otros sistemas intrarradiculares.

Los ítems del cuestionario fueron escritos en un lenguaje sencillo, evitando términos técnicos y sujetos a un consenso de expertos para validar su contenido y constructo, realizándose una prueba piloto antes de su aplicación final.

Se realizó un análisis descriptivo para variables tales como: el efecto férula, empleo de radiografía, la influencia del costo, postes utilizados en el sector anterior y posterior. Frecuencias para variables categóricas e intervalos de confianza del $95 \%$ (IC). Se realizó un análisis de estadística inferencial para establecer la relación entre el uso de los distintos criterios elegidos, frente a respuestas apropiadas para su uso, utilizando la prueba del chi cuadrado por ser variables cualitativas. El análisis estadístico se realizó con SPSS 20.0 (IBM, EE.UU.). En todos los casos p <0,05 se consideró significativo.

\section{RESULTADOS}

En el análisis bivariado en la tabla 1 se identificó criterios de uso en relación a la técnica de obtención y el material $(\mathrm{p}=0,00)$, la ausencia de estructura dental remanente $(\mathrm{p}=0,00)$; asociados con la frecuencia de su aplicación. En la tabla 2 se identifica la experiencia de fractura $(\mathrm{p}=0,348)$, el conocimiento de otros sistemas de postes intrarradiculares $(\mathrm{p}=0,00)$; asociado con la experiencia clínica y conocimiento. En la tabla 3 se identificó el sistema de cementación para 


\begin{tabular}{|c|c|c|c|c|c|c|}
\hline Caracteristica & & $\begin{array}{c}\text { Siempre } \\
\mathrm{n}(\%)\end{array}$ & $\begin{array}{c}\text { Algunas veces } \\
\mathrm{n}(\%)\end{array}$ & $\begin{array}{l}\text { Nunca } \\
\mathrm{n}(\%)\end{array}$ & $\mathrm{x}^{2}$ & $\mathrm{p}$ \\
\hline $\begin{array}{l}\text { Ténnica de } \\
\text { obtención y }\end{array}$ & $\begin{array}{c}\text { Prefabricado } \\
\text { (fibra de vidrio) }\end{array}$ & $19(19,8)$ & $65(65,7)$ & $12(12,5)$ & 5,1 & 0,00 \\
\hline material & $\begin{array}{l}\text { Metálico } \\
\text { (colados) }\end{array}$ & $68(68.8)$ & $30(31,3)$ & & & \\
\hline $\begin{array}{l}\text { Ausencia de } \\
\text { estructura }\end{array}$ & $\begin{array}{c}\text { Prefabricado } \\
\text { (fibra de vidrio) }\end{array}$ & $4(4,3)$ & $33(35,1)$ & $57(60,6)$ & 18,4 & 0,00 \\
\hline $\begin{array}{l}\text { coronal } \\
\text { remanente }\end{array}$ & $\begin{array}{l}\text { Metálico } \\
\text { (colados) }\end{array}$ & $88(93,6)$ & $(6,4)$ & & & \\
\hline
\end{tabular}

Tabla 2. Experiencia de fractura, conocimiento de sistemas

\begin{tabular}{|c|c|c|c|c|c|}
\hline \multirow[t]{2}{*}{ Característica } & & $\mathrm{Si}$ & No & $\mathrm{x}^{2}$ & $\mathrm{p}$ \\
\hline & & $\mathrm{n}(\%)$ & $\mathrm{n}(\%)$ & \multirow{3}{*}{0,88} & \multirow{3}{*}{0,348} \\
\hline $\begin{array}{l}\text { Experiencia de } \\
\text { fractura }\end{array}$ & $\begin{array}{c}\text { Prefabricado } \\
\text { (fibra de vidrio) }\end{array}$ & $14(14,1)$ & $85(85,9)$ & & \\
\hline \multirow{3}{*}{$\begin{array}{l}\text { Conocimiento } \\
\text { de sistemas }\end{array}$} & $\begin{array}{l}\text { Metálico } \\
\text { (colados) }\end{array}$ & $24(24.2)$ & $75(75,8)$ & & \\
\hline & Odontólogo & $8(8,0)$ & $60(60,0)$ & \multirow{2}{*}{12,9} & \multirow{2}{*}{0,0} \\
\hline & Especialistas & $14(14,0)$ & $18(18,0)$ & & \\
\hline
\end{tabular}

los postes intrarradiculares $(\mathrm{p}=0,00)$, asociado con los diferentes tipos de cementos. Dentro de los cuales encontramos significancia estadística y dependencia de las variables entre sí. En la tabla 4 muestra las características de frecuencia de utilización del efecto férula, el empleo de radiografía previa y la influencia del costo. En las tablas 5 y 6 muestra el tipo de restauración utilizada en el sector anterior y posterior según la clasificación de Murgueito. ${ }^{13}$

\begin{tabular}{|c|c|c|c|c|c|c|c|}
\hline & & & \multicolumn{4}{|c|}{ PREF. (FIIRA } & \multirow[b]{2}{*}{ Total } \\
\hline & & & $\begin{array}{l}\text { POLICARBOXILA } \\
\text { TO DE ZINC }\end{array}$ & $\begin{array}{l}\text { IONÓMERO } \\
\text { DE VIDRIO }\end{array}$ & $\begin{array}{l}\text { CEMENTO } \\
\text { RESINOSO }\end{array}$ & $\begin{array}{c}\text { CEMENTO } \\
\text { DUAL }\end{array}$ & \\
\hline & \multirow{2}{*}{$\begin{array}{l}\text { FOSFATO DE } \\
\text { ZINC }\end{array}$} & Recuento & 0 & 17 & 1 & 10 & 28 \\
\hline & & $\%$ del total & 0,0 & 18,9 & 1,1 & 11,1 & $31,1 \%$ \\
\hline & \multirow{2}{*}{$\begin{array}{l}\text { POLICARBOXILA } \\
\text { TO DE ZINC }\end{array}$} & Recuento & 0 & 3 & 4 & 2 & 9 \\
\hline \multirow{4}{*}{$\begin{array}{l}\text { METÁAICOS } \\
\text { (COLADOS) }\end{array}$} & & $\%$ del total & 0,0 & 3,3 & 4,4 & 2,2 & $10,0 \%$ \\
\hline & \multirow{2}{*}{$\begin{array}{l}\text { IONÓMERO DE } \\
\text { VIDRIO }\end{array}$} & Recuento & 1 & 10 & 2 & 39 & 52 \\
\hline & & $\%$ del total & 1,1 & 11,1 & 2,2 & 43,3 & $57,8 \%$ \\
\hline & \multirow[t]{2}{*}{ CEMENTO DUAL } & Recuento & 0 & 0 & 0 & 1 & 1 \\
\hline & & $\%$ del total & 0,0 & 0,0 & 0,0 & 1,1 & $1,1 \%$ \\
\hline \multirow[t]{2}{*}{ Total } & & Recuento & 1 & 30 & 7 & 52 & 90 \\
\hline & & $\%$ del total & $1,1 \%$ & $33,3 \%$ & $7,8 \%$ & $57,8 \%$ & $100,0 \%$ \\
\hline
\end{tabular}

Tabla 4. Efecto férula, empleo de radiografía e influencia del costo.

\begin{tabular}{ccccc}
\hline & $\begin{array}{c}\text { SIEMPRE } \\
\mathrm{f} \%\end{array}$ & $\begin{array}{c}\text { ALGUNAS } \\
\text { VECES } \\
\mathrm{f} \%\end{array}$ & $\begin{array}{c}\text { NUNCA } \\
\mathrm{f} \%\end{array}$ & TOTAL \\
\hline $\begin{array}{c}\text { Efecto férula } \\
(2 \mathrm{~mm})\end{array}$ & 38 & 61 & 1 & 100 \\
$\begin{array}{c}\text { Empleo de } \\
\text { radiografia }\end{array}$ & 83 & 17 & & 100 \\
$\begin{array}{c}\text { Influencia del } \\
\text { costo }\end{array}$ & 35 & 53 & 12 & 100 \\
\hline Fuente: Matriz de sistematización & & &
\end{tabular}

Tabla 5. Tipo de restauración utilizada en el sector anterior

\begin{tabular}{ccccc}
\hline & $\begin{array}{c}\text { CLASE I } \\
\text { f } \%\end{array}$ & $\begin{array}{c}\text { CLASE II } \\
\text { f } \%\end{array}$ & CLASE III & CLASE IV \\
\hline Poste colado con NPG & 1 & 5 & 44 & 60 \\
Poste colado en Verabond & 1 & 4 & 17 & 25 \\
Poste colado en Verasoft & & 1 & 10 & 14 \\
Poste de fibra de vidrio & 6 & 34 & 27 & 1 \\
Poste prefabricado metálico & 1 & 6 & 2 & \\
Restauración directa & 91 & 50 & & \\
TOTAL & 100 & 100 & 100 & 100 \\
\hline Fuente: Matriz de sistematización & & &
\end{tabular}

Tabla 6. Tipo de restauración utilizada en el sector posterior

\begin{tabular}{cccccc}
\hline & CLASE I & $\begin{array}{c}\text { CLASE } \\
\text { II-A } \\
\text { f } \%\end{array}$ & $\begin{array}{c}\text { CLASE } \\
\text { II-B } \\
\text { f } \%\end{array}$ & $\begin{array}{c}\text { CLASE } \\
\text { III } \%\end{array}$ & $\begin{array}{c}\text { CLASE } \\
\text { f } \%\end{array}$ \\
\hline $\begin{array}{c}\text { Poste colado con } \\
\text { NPG }\end{array}$ & 1 & 4 & 10 & 13 & 14 \\
$\begin{array}{c}\text { Poste colado en } \\
\text { Verabond }\end{array}$ & 4 & 12 & 42 & 58 & 59 \\
$\begin{array}{c}\text { Poste colado en } \\
\text { Verasoft }\end{array}$ & 1 & 3 & 11 & 23 & 26 \\
$\begin{array}{c}\text { Poste de fibra de } \\
\text { vidrio }\end{array}$ & 4 & 8 & 17 & 4 & 1 \\
$\begin{array}{c}\text { Poste } \\
\text { prefabricado } \\
\text { metálico }\end{array}$ & 2 & 6 & 3 & 2 & 0 \\
$\begin{array}{c}\text { Restauración } \\
\text { directa o indirecta } \\
\text { TOTAL }\end{array}$ & 88 & 67 & 17 & 0 & 0 \\
\hline Fuente: Matriz de sistematización & 100 & 100 & 100 & 100 \\
\hline
\end{tabular}

\section{DISCUSIÓN}

En este estudio la encuesta realizada entre los odontólogos de la ciudad de Juliaca para analizar las preferencias durante los aspectos en el uso de postes intrarradiculares, tuvo hallazgos importantes; ya que la encuesta basada en cuestionario proporciona información relevante en la percepción, actitud y enfoque hacia el tratamiento. El estudio nos muestra que los odontólogos de la ciudad de Juliaca tienen una mayor preferencia hacia los postes metálicos colados, tanto en los criterios de ausencia de estructura coronal remanente, 73,6\% siempre los prefiere, como para la forma de obtención y el material $68,8 \%$ siempre los prefiere, siendo de mayor preferencia frente a los postes de fibra de vidrio. Ahmed et al., en su estudio encontraron la preferencia en los postes de fibra de vidrio $72 \%$, seguido de las aleaciones prefabricadas (38,6\%), postes de metal fundido $33,9 \%$, postes prefabricados de titanio $30,1 \%$, y los postes de acero inoxidable $21,7 \% .^{14}$ En otro estudio realizado por Sarkis et al., encontraron que un $24,53 \%$ eligió el poste metálico colado, un $20,75 \%$ para los postes de fibra de vidrio, ${ }^{15}$ siendo datos estadísticos asimilables a nuestro estudio. Por lo que en ambos estudios tiene mayor importancia el uso de postes de fibra de vidrio por la mayor innovación y acceso que presenta a los 
materiales; contrario a nuestra región en la que no se tiene un acceso en su totalidad a los materiales que son tendencia a nivel mundial.

Para la rehabilitación del sector anterior según la clasificación de Murgueito se encontró que en una clase I, un $91 \%$ prefiere la restauración directa, en tanto para la clase II un $50 \%$ indica la restauración directa y un (34\%) el uso de poste de fibra de vidrio; en una clase III un $44 \%$ indica la utilización de un poste colado en NPG, un $27 \%$ el uso de poste de fibra de vidrio y en una clase IV un $60 \%$ manifestó el uso de un poste colado en NPG. ${ }^{13}$ En el sector posterior se encontró que para una clase I un $88 \%$ manifiesta el uso de una restauración, en una clase II-A un $67 \%$ manifiesta la restauración, para la clase II-B un $42 \%$ aplica la rehabilitación con poste colado en Verabond, $17 \%$ el uso de poste de fibra de vidrio, en una clase III un $58 \%$ considera la rehabilitación con un poste colado en verabond y en la clase IV un $59 \%$ considera la rehabilitación con un poste colado en verabond; la restauración fue considerada ya sea directa e indirecta dependiendo del criterio clínico de cada profesional. En un estudio realizado por Bolla et al., no pudo especificar qué tipo de sistema de pernos debe usarse cuando dos o tres paredes de dentina se mantienen, por lo que manifiesta el uso de más ensayos clínicos para confirmar que sistemas de pernos muñones reforzados con fibra son superiores y para determinar la influencia de la estructura dental remanente en el resultado del tratamiento de los diferentes sistemas de perno muñón disponibles. ${ }^{16}$ Deben usarse criterios de inclusión bien definidos que se centran en el número de paredes de dentina (dos o tres). La decisión se basa en la cantidad de estructura remanente y al tipo de diente, por lo que en nuestro estudio se clasifica los usos de los distintitos tipos de postes tanto para el sector anterior como el sector posterior, según la clasificación de Murgueito con presencia de más de tres paredes, teniendo resultados favorables y aceptados estadísticamente. ${ }^{13}$ En el estudio de Kon la mayoría de los encuestados en cuanto al tipo de postes, utilizaron predominantemente postes metálicos, mientras que se registró una aplicación cada vez mayor de postes de fibra de vidrio. ${ }^{17}$ Contrastando con nuestro estudio ya que la educación continua influye en la decisión de los dentistas en su elección de los postes dentales a ser utilizados.

En cuanto a la consideración del criterio del efecto férula de $2 \mathrm{~mm}$ un $61 \%$ lo considera algunas veces mientras que un $38 \%$ lo considera siempre, por lo que difiere de otros estudios en los cuales el éxito de la rehabilitación se basa en la consideración del efecto férula para una mejor rehabilitación post endodoncia y en nuestro estudio solo es considerado algunas veces. La cantidad residual de la estructura dental determinará su estabilidad para la restauración. La creación de la férula adecuada (se acerca a $2 \mathrm{~mm}$ circunferencialmente es lo ideal) minimiza los efectos perjudiciales de las fuerzas laterales y de rotación en la posterior restauración. ${ }^{18}$ En otro estudio el efecto de férula fue considerado como un factor clave de éxito se llevó a cabo por la inmensa mayoría de los dentistas $88 \% .{ }^{19}$ Además, se ha demostrado que la presencia de la férula (mínimo 2 $\mathrm{mm}$ ) es un factor decisivo para el éxito del pilar fundido y el núcleo. ${ }^{20}$

El estudio muestra una fuerte incidencia en el criterio de la influencia del costo, ya que un $53 \%$ indica que el costo influye a la hora de elegir un tratamiento rehabilitador de acorde a los avances científicos. La población estudiada tiene un $78 \%$ de desconocimiento de otro tipo de sistemas de rehabilitación post endodóntica. En lo que se refiere al empleo de radiografía previa a la rehabilitación, un $83 \%$ siempre lo considera de vital importancia previo al tratamiento rehabilitador.

En lo que se refiere al criterio de cementación, en el estudio se observó que para la cementación de postes intrarradiculares metálicos colados un $57,8 \%$ manifiesta la cementación con ionómero de vidrio, seguido de un $31,1 \%$ que realiza la cementación con fosfato de zinc; para la cementación de postes de fibra de vidrio un $(57 \%)$ considera la cementación con el cemento dual, seguido de un $33,3 \%$ que emplea el ionómero de vidrio. En otro estudio la cementación con resinas compuestas $49 \%$ tiene mayor afluencia para ser utilizado, dos veces más que el cemento de fosfato de zinc $24 \% .{ }^{19}$ En la última década, se introdujeron cementos de resina autoadhesivos para proporcionar la aplicación clínica más fácil, en comparación con los cementos de resina regulares. ${ }^{21}$ Una reciente revisión sistemática de los estudios in vitro mostró que la literatura sobre estos estudios sugiere que el cemento de resina autoadhesivo podría mejorar la retención de los puntos focales de género en los conductos radiculares. ${ }^{22}$ En otro estudio muestra que las técnicas de aplicación y manejo pueden influir en la resistencia de la unión de diferentes cementos autoadhesivas cuando se utiliza para la cementación de postes intrarradiculares. ${ }^{23}$ Sin embargo, una limitación importante de este estudio se relaciona con la cuestión de la elección del cemento, ya que no se le dio la opción de cemento de fosfato de zinc. Esto debe hacerse hincapié, ya que los cementos de fosfato de zinc son ampliamente utilizados, especialmente para cementar postes de metal fundido, debido a su larga historia de éxito, así como su precio más bajo y su técnica menos sensible, en comparación con los cementos de resina. ${ }^{24}$

La literatura sugiere que endocoronas pueden llevar a cabo de manera similar o mejor que los tratamientos convencionales que utilizan postes intrarradiculares, restauraciones con resina compuesta directa o inlay / onlay. ${ }^{25}$ 
El éxito de la restauración de un diente tratado endodónticamente es un desafío permanente para un dentista restaurador. Antes de insertar un poste, factores como la cantidad restante de tejido coronal, tamaño de la raíz, configuración del canal, posición de los dientes, los requisitos funcionales y la oclusión deben ser analizados. ${ }^{26}$

Este estudio tuvo algunas limitaciones, ya que se basó en un cuestionario cerrado auto-aplicado. Algunas desventajas incluyen el hecho de que la auto-aplicación requiere otra visita por el personal de investigación, y el entrevistado no siempre contesta todas las preguntas del cuestionario.

\section{Referencias}

1 DE Oliveira BP, Aguiar CM, Câmara AC, DE Albuquerque MM, Correia AC, Soares MF. Evaluation of microbial reduction in root canals instrumented with reciprocating and rotary systems. Acta Stomatol Croat. 2015;49(4):294-303.

2 Rubio J, Zarzosa JI, Pallarés A. A comparative study of shaping ability of four rotary systems. Acta Stomatol Croat. 2015;49(4):285-93.

3 Monterde M, Pallarés A, C Cabanillas, Zarzosa I, Victoria A. A Comparative in Vitro Study of Apical Microleakage with Five Obturation Techniques: Lateral Condensation, Soft-Core $\mathrm{R}$, Obtura II $\mathrm{R}$, Guttaflow $\mathrm{R}$ and Resilon $\mathrm{R}$. Acta Stomatol Croat. 2014;48(2):123-31.

4 Ozbay G, B Kitiki, Peker S, Kargul B. Apical Sealing Ability of a Novel Material: Analysis by Fluid Filtration Technique. Acta Stomatol Croat. 2014;48(2):132-9

5 Gillen BM, Looney SW, Gu LS, et al. Impact of the quality of coronal restoration versus the quality of root canal fillings on success of root canal treatment: a systematic review and meta-analysis. J Endod. 2011;37(7):895-902.

6 Christensen GJ. Intracoronal and extracoronal tooth restorations 1999. J Am Dent Assoc. 1999;130(4):557-60.

7 Coelho MDSC, Biffi JCG, Da Silva GR, Abrahão A, Campos RE, Soares CJ . Finite element analysis of weakened roots restored with composite resin and posts. Dent Mater J. 2009;28(6):671-8.

8 Dietschi D, Duc O, Krejci I, Sadan A. Biomechanical considerations for the restoration of endodontically treated teeth: a systematic review of the literature. Part II (Evaluation of fatigue behavior, interfaces, and in vivo studies). Quintessence Int 2008;39:117-29.

9 Schwartz RS, Robbins JW. Post placement and restoration of endodontically treated teeth: a literature review. J Endod. 2004;30:289-301.

10 Horn SD, Gassaway J. Practice-based evidence study design for comparative effectiveness research. Med Care. 2007;45(10):50-7.

11 Opdam NJ, Roeters JJ, Loomans BA, Bronkhorst EM. Sevenyear clinical evaluation of painful cracked teeth restored with a direct composite restoration. J Endod. 2008;34(7):808-11.

2 Gilbert GH, Richman JS, Gordan VV, et al. DPBRN Collaborative Group. Lessons learned during the conduct of clinical studies in the dental PBRN. J Dent Educ. 2011;75(4):453-65.

13 Murgueitio R. Classification of the extension defects of endodontically treated posterior teeth. Rev Estomat. 2008;16(2):31-37.

4 Ahmed SN, Donovan TE, Ghuman T. Survey of dentists to determine contemporary use of endodontic posts. J Prosthet Dent. 2017;117(5):642-55.

15 Sarkis-Onofre R, Pereira-Cenci T, Opdam NJ, Demarco FF. Preference for using posts to restore endodontically treated teeth: findings from a survey with dentists. Braz Oral Res. 2015;29:1-6.

16 Bolla M, Muller-Bolla M, Borg C, Lupi-Pegurier L, Laplanche O, Leforestier E. WITHDRAWN: Root canal posts for the restoration of root filled teeth. Cochrane Database Syst Rev. 2016;11(11):CD004623.

17 Kon M, Zitzmann NU, Weiger R, Krastl G. Postendodontic restoration: a survey among dentists in Switzerland. Schweiz Monatsschr Zahnmed. 2013;123(12):1076-88.

18 Trushkowsky RD. Restoration of endodontically treated teeth: criteria and technique considerations. Quintessence Int. 2014;45(7):557-67

19 Naumann M, Neuhaus KW, Kölpin M, Seemann R. Why, when, and how general practitioners restore endodontically treated teeth: a representative survey in Germany. Clin Oral Investig. 2016;20(2):253-9.

20 Silva NR, Raposo LH, Versluis A, Fernandes-Neto AJ, Soares CJ. The effect of post, core, crown type, and ferrule presence on the biomechanical behavior of endodontically treated bovine anterior teeth. J Prosthet Dent. 2010;104(5):30617.

21 Ferracane JL, Stansbury JW, Burke FJ. Self-adhesive resin cements-Chemistry, properties and clinical considerations. J Oral Rehabil. 2011:38(4);295-14.

22 Sarkis-Onofre R, Skupien JA, Cenci MS, Moraes RR, Pereira-Cenci T. The role of resin cement on bond strength of glass-fiber posts luted into root canals: a systematic review and meta-analysis of in vitro studies. Oper Dent. 2014; 39(1):31-44.

23 Shiratori FK, Valle AL, Pegoraro TA, Carvalho RM, Pereira JR. Influence of technique and manipulation on selfadhesive resin cements used to cement intraradicular posts. J Prosthet Dent. 2013;110(1):56-60.

24 Farid F, Mahgoli H, Hosseini A, Chiniforush N. Effect of eugenol-containing and resin endodontic sealers on retention of prefabricated metal posts cemented with zinc phosphate and resin cements. J Prosthodont Res. 2013;57(4):28487.

25 Sedrez-Porto JA, Rosa WL, da Silva AF, Münchow EA, Pereira-Cenci T. Endocrown restorations: A systematic re- 
view and meta-analysis. J Dent. 2016;52:8-14.

26 Parčina I, Amižić, Baraba A. Esthetic Intracanal Posts. Acta Stomatol Croat. 2016;50(2):143-50.

Recibido: 21 de enero de 2020

Aceptado: 2 de abril de 2020 